\title{
MORTALIDADE BRASILEIRA POR CÂNCER DE ESÔFAGO NOS ÚLTIMOS 10 ANOS
}

\section{ARTIGO ORIGINAL}

LEITE, Renata Stefanny Alves ${ }^{1}$, SILVA, Rafael Luiz do Rego²

LEITE, Renata Stefanny Alves. SILVA, Rafael Luiz do Rego. Mortalidade brasileira por câncer de esôfago nos últimos 10 anos. Revista Científica Multidisciplinar Núcleo do Conhecimento. Ano 06, Ed. 08, Vol. 06, pp. 19-26. Agosto de 2021. ISSN: 2448-0959, Link de acesso:

https://www.nucleodoconhecimento.com.br/saude/mortalidade-brasileira, DOI: 10.32749/nucleodoconhecimento.com.br/saude/mortalidade-brasileira

\section{RESUMO}

Introdução: O câncer de esôfago é uma neoplasia bastante agressiva que está entre os dez mais incidentes no país e o sexto quando se relaciona a mortalidade por câncer. O Carcinoma epidermóide é o tipo histológico mais prevalente e está relacionado a fatores de riscos como tabagismo, etilismo, dietas a base de produtos industrializados ricos em nitrosaminas. Objetivo: Caracterizar o perfil de mortalidade por câncer de esôfago no Brasil. Método: Estudo transversal, descritivo e retrospectivo, em que foi utilizado as bases de dados do Sistema de informação Hospitalares do SUS (SIH/SUS) alimentada pela Autorização de Internação Hospitalar (AIH) e pelo Sistema de Informações sobre a Mortalidade (SIM), ambos disponíveis no Departamento de Informática do Sistema Único de Saúde (DATASUS). Resultados e discussões: O número de óbitos no período em estudo foi de 30.171 casos, sendo a taxa de letalidade hospitalar $16,00 \%$. Com relação à faixa etária, o número de óbitos foi maior entre os 50 aos 69 anos (58,69\%), seguida pela faixa etária dos 70 aos 79 anos $(20,16 \%)$. Entre os sexos, o masculino foi

\footnotetext{
${ }^{1}$ Graduação em medicina na Universidade Federal de Alagoas.

${ }^{2}$ Acadêmico de Medicina da Universidade Maurício de Nassau.
}

RC: 95785

Disponível em: https://www.nucleodoconhecimento.com.br/saude/mortalidadebrasileira 
significativamente maior $(77,33 \%)$ e entre as regiões, o sudeste detém mais da metade no número de óbitos $(51,73 \%)$. Este estudo demonstrou alta associação da neoplasia com o sexo masculino. Apesar do número de óbitos ter sido maior na região sudeste, a região norte foi a que apresentou maior taxa de mortalidade por câncer de esôfago. Conclusão: A importância de se investimentos na área para que tanto o acesso ao diagnóstico seja realizado de maneira precoce, como o tratamento reduza a taxa de desfechos desfavoráveis. Além disso, medidas de conscientização social ainda na atenção primária a respeito das condições que podem predispor o câncer de esôfago, alertando a população a respeito de fatores ainda pouco conhecidos relacionados.

Palavras-Chave: Câncer de Esôfago/Epidemiologia, Câncer de Esôfago/ Mortalidade, DATASUS, Hospitalização.

\section{INTRODUÇÃO}

O câncer de esôfago configura-se como uma neoplasia bastante agressiva que, segundo dados do INCA, está entre os dez mais incidentes no país e o sexto quando se relaciona a mortalidade por câncer. Além disso, foram estimados 11.390 novos casos no ano de 2020 no país, sendo 8.690 homens e 2.700 mulheres e computou-se 8.716 óbitos no ano de 2019 (INCA, 2021).

Dentre os tipos histológicos encontrados, o Carcinoma epidermóide, oriundo do epitélio estratificado não-queratinizado da própria mucosa do órgão, é o mais prevalente e está muito relacionado a fatores de riscos como o tabagismo, etilismo, dietas a base de produtos industrializados ricos em nitrosaminas (BATRA, 2019). Este tipo histológico costuma ocupar a região de terço superior do esôfago (ALSOP, 2016). Já o adenocarcinoma está mais localizado na região distal, sendo relacionado a metaplasia intestinal no esôfago de Barret, condição correlacionada com a esofagite causada pelo refluxo gastroesofágico (NAINI, 2016). 
Em relação ao quadro clínico do paciente, têm-se os sintomas da disfagia ocorrendo de forma mais recente e progressiva, iniciando-se por alimentos sólidos e seguindo posteriormente para líquidos (ZETERKA, 2016).

Junto a isso, a perda ponderal de $10 \%$ três a seis meses se fazem pontos chaves na suspeita do câncer que, na maioria das vezes, são sintomas que representam uma fase já avançada, já que estágios iniciais costumam ser assintomáticos. Outros sintomas incluem odinofagia, dor torácica, náuseas, vômitos e perda de apetite (SHORT, 2017). A busca pelo diagnóstico correto é feita a partir da esofagografia baritada que mostram sinais típicos como o "sinal da maçã mordida" e posteriormente é confirmado pela Endoscopia digestiva alta a partir da biópsia (SABISTON, 2010).

Infelizmente, estágios iniciais são raros de ser identificados, e por isso, o prognóstico para o câncer de esôfago permanece sombrio, com taxas de sobrevida global inferior a 20\% em cinco anos (VENDRELY, 2018).

Nesse sentido, cabe analisar a mortalidade brasileira por neoplasias de esôfago no Brasil nos últimos 10 anos a fim de se estabelecer um perfil epidemiológico dessa patologia.

Objetivo geral: Caracterizar o perfil de mortalidade por câncer de esôfago no Brasil.

Objetivos específicos: Identificar o número de óbitos e o de internamentos em decorrência do câncer de esôfago. Avaliar a prevalência entre as regiões brasileira desta patologia; Caracterizar o perfil epidemiológico das mortes e dos internamentos de acordo com a idade, sexo e etnia; Estabelecer o tempo de permanência hospitalar dos pacientes dos portadores deste câncer.

\section{MÉTODOS}

Trata-se de um estudo transversal, descritivo e retrospectivo, em que foi utilizado as bases de dados do Sistema de informação Hospitalares do SUS (SIH/SUS) 
alimentada pela Autorização de Internação Hospitalar (AIH) e pelo Sistema de Informações sobre a Mortalidade (SIM), ambos disponíveis no Departamento de Informática do Sistema Único de Saúde (DATASUS) no endereço eletrônico <http://www2.datasus.gov.br/DATASUS/index.php?area=0203\&id=6926\&VObj=http:/ /tabnet.datasus.gov.br/cgi/deftohtm.exe?sih/cnv/ni.> acessado no dia 01 de maio de 2021.

O período em análise compreende-se de janeiro de 2010 a dezembro de 2020. As variáveis utilizadas pelo estudo foram: número de internamentos, número de óbitos e taxa de mortalidade estratificados por faixa etária, região, etnia (cor/raça) e sexo dos pacientes internados com diagnóstico primário de neoplasia maligna do esôfago. Observou-se, também, o tempo médio de permanência hospitalar em dias. Para o cálculo da taxa de mortalidade hospitalar, foi utilizada a razão entre o número de óbitos e o de pacientes internados. O programa utilizado para construir as tabelas foi o Excel da Microsoft巴

\section{RESULTADOS}

De janeiro de 2010 a dezembro de 2020 foram computados 188.503 internamentos por neoplasia maligna de esôfago em todo território nacional (17.136 internamentos/ano), sendo a região sudeste a que apresentou o maior número de internamentos: 49,58\% no período analisado. Houve maior número de internamentos entre o sexo masculino (76,64\%) na faixa etária dos 50 aos 69 anos (61,36\% - Gráfico 1) e em pacientes da cor/raça branca (40,77\%) seguidos da raça/cor parda (34,81\%). O número de internamentos se tornou progressivamente maior no decorrer dos anos, porém no último ano analisado houve uma redução de $15,4 \%$ em relação ao ano anterior. 
Gráfico 1: Número de internamento por faixa etária

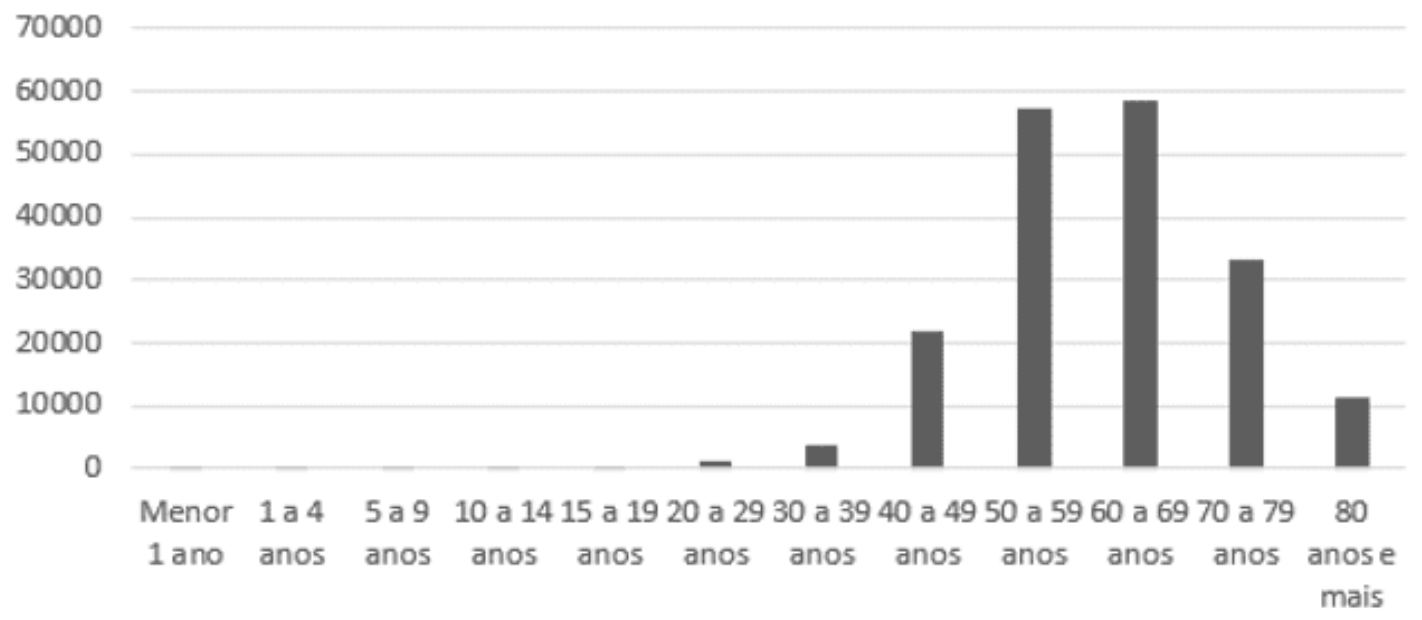

Fonte: os autores.

O número de óbitos no período foi de 30.171 casos, sendo a taxa de letalidade hospitalar 16,00\% (número de óbitos pelo número de internamentos). Com relação à faixa etária, o número de óbitos foi maior entre os 50 aos 69 anos ( $58,69 \%$ do total de óbitos), seguida pela faixa etária dos 70 aos 79 anos (20,16\%). Já entre os sexos, o masculino foi significativamente maior $(77,33 \%$ - Gráfico 3$)$ e entre as regiões, o sudeste detém mais da metade no número de óbitos (51,73\% - Gráfico 2).

RC: 95785

Disponível em: https://www.nucleodoconhecimento.com.br/saude/mortalidadebrasileira 
Gráfico 2: Número de óbitos por região brasileira

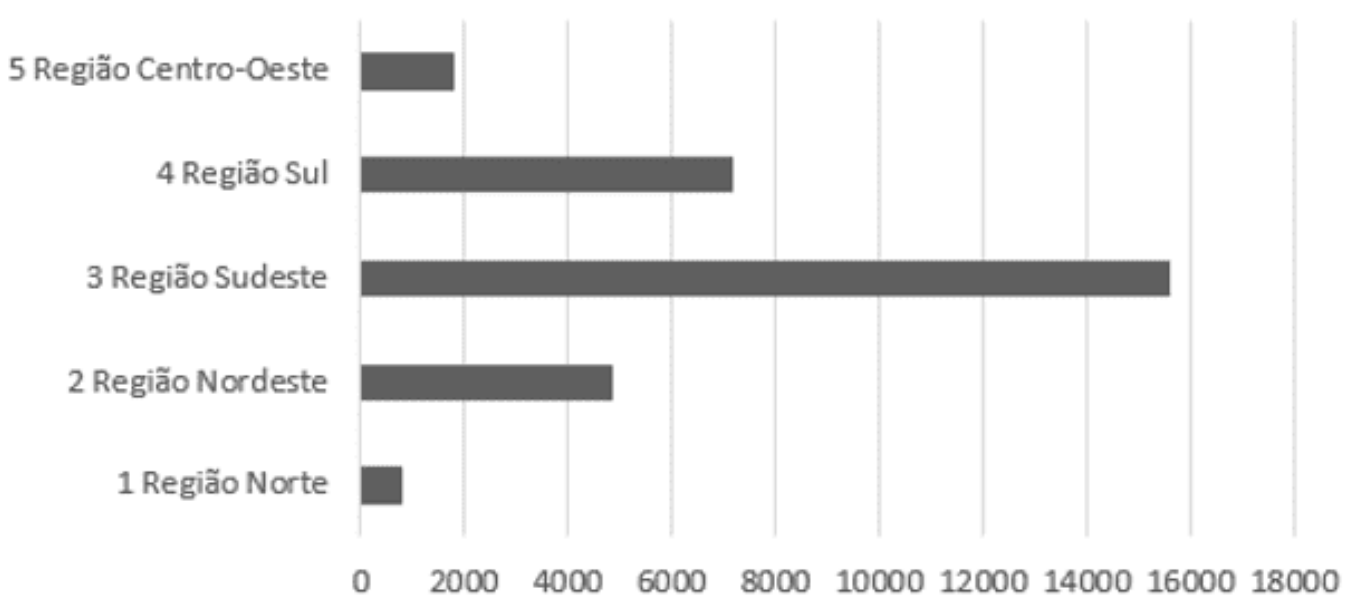

Fonte: os autores.

A média de permanência hospitalar foi de 7,0 dias, sendo igual entre os sexos. Com relação às faixas etárias e as regiões, o tempo de permanência hospitalar não apresentou grandes variações.

A taxa de mortalidade geral no período analisado foi de 16,01 a cada 10.000 habitantes e apresentou pequenas variações, sendo a maior em 2015, com 16,9. A região Norte foi a de maior taxa de mortalidade $(19,29)$. A taxa de mortalidade específica por sexo foi de 16,15 para os homens e de 15,54 para as mulheres (Gráfico 3) e por faixa etária notou-se um aumento progressivo da taxa de mortalidade quanto mais velho o grupo populacional observado, sendo a maior taxa nos pacientes acima de 80 anos (24,61 em 10. 000 habitantes)

RC: 95785

Disponível em: https://www.nucleodoconhecimento.com.br/saude/mortalidadebrasileira 
Gráfico 3: óbitos por sexo

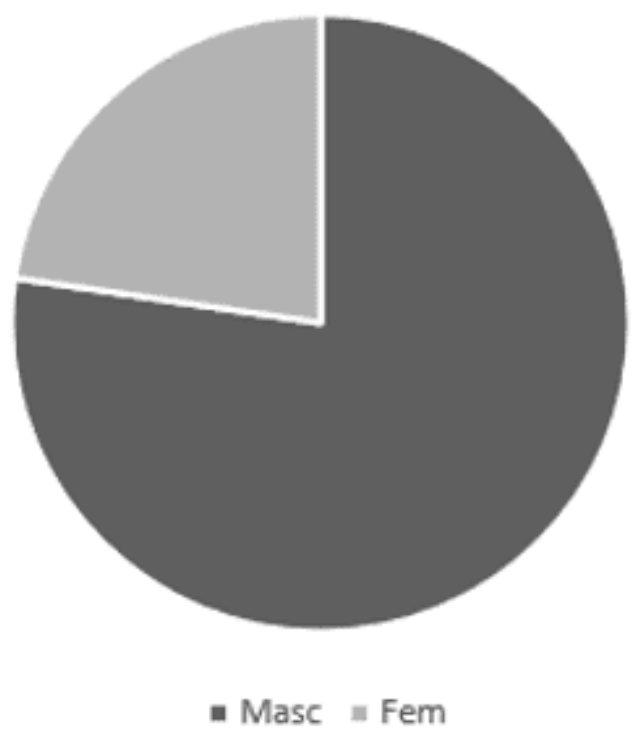

Fonte: os autores.

\section{DISCUSSÃO}

Segundo dados do Instituto Nacional de Câncer (INCA) o câncer de esôfago é duas vezes mais prevalente em homens do que em mulheres e este estudo, de fato, demonstrou alta associação da neoplasia com o sexo masculino. Nota-se ainda que, apesar do número de óbitos ter sido maior na região sudeste, a região norte foi a que apresentou maior taxa de mortalidade por câncer de esôfago. Ressalta-se também que a raça branca obteve os piores parâmetros em todas as variáveis observadas.

Não há evidência científica de que o rastreamento do câncer de esôfago traga mais benefícios do que riscos e, portanto, até o momento, ele não é recomendado (INCA, 2021). 


\section{CONCLUSÕES}

Com isso, nota-se que no Brasil existe altos índices de mortalidade em decorrência do câncer de esôfago e, por isso, é importante que haja investimentos na área para que tanto o acesso ao diagnóstico seja realizado de maneira precoce, como o tratamento reduza a taxa de desfechos desfavoráveis. Além disso medidas de conscientização social ainda na atenção primária a respeito das condições que podem predispor o câncer de esôfago, principalmente alertando-se a população a respeito de fatores ainda pouco conhecidos relacionados à mudança no perfil epidemiológico ocorrida no Brasil. Dentre esses fatores, a mudança no perfil alimentar entra como uma importante estratégia na prevenção, já que a obesidade tem se tornado um "carro chefe" tanto para predisposição quanto para prognóstico dessa neoplasia. É imprescindível colocar em prática o princípio organizacional de hierarquização dos SUS que envolvem toda uma base de cuidados de pequenas complexidades para que a partir da identificação de populações de risco, possa-se referenciar e consequentemente instituir possíveis abordagens terapêuticas eficazes.

Por fim, a partir desse estudo, inspiramos a criação de novos delineamentos investigativos de cunho analítico e individualizado que torne possível entender a real associação dos tumores malignos de esôfago com o sexo masculino, por exemplo.

\section{REFERÊNCIAS}

ALSOP, B. R; SHARMA P. Esophageal Cancer. Gastroenterol. Clin North Am. Vol 45.Pág 399-412. September 2016

BATRA R, et al Managing Squamous Cell Esophageal Cancer. Surg Clin North Am. Vol 99. Pág 529-541. Jun, 2019 
BRASIL. Instituto Nacional do Câncer - Ministério da Saúde. Câncer de Esôfago. Disponível em: https:/www.inca.gov.br/tipos-de-cancer/cancer-de-esofago Acessado 01 de maio de 2021

BRASIL. Ministério da Saúde Disponível em: ttp://www2.datasus.gov.br/DATASUS/index.php?area=0203\&id=6926\&VObj=htt p://tabnet.datasus.gov.br/cgi/deftohtm.exe?sih/cnv/ni Acessado no dia 01 de maio de 2021.

NAINI, B. V; SOUZA; R. F; ODZE R. D. Barrett's Esophagus: A Comprehensive and Contemporary Review for Pathologists. Am J Surg Pathol Rockville Pike. Vol 40. Pag 45-66. Maio de 2016.

SABISTON, et al. Sabiston Tratado de cirurgia. A base biológica da prática cirúrgica Moderna. 19a edição. Rio de Janeiro. Elsevier. 2010.

VENDRELY V, et al. Prognostic factors in esophageal cancer treated with curative intent. Dig Liver Dis: Official Journal of the Italian Society of Gastroenterology and the ItalianAssociation for the Study of the Liver. Vol 50. Pág 991-996. Out 2018

ZETERKA, S; EISING, J. N. Tratado de gastroenterologia : da graduação à pósgraduação. São Paulo: Editora Atheneu, 2016, 2. edição

Enviado: Março, 2021.

Aprovado: Agosto, 2021.

RC: 95785

Disponível em: https://www.nucleodoconhecimento.com.br/saude/mortalidadebrasileira 